\title{
Production of biologically active phenolic acids in Aronia melanocarpa (Michx.) Elliott in vitro cultures cultivated on different variants of the Murashige and Skoog medium
}

\author{
Agnieszka Szopa • Halina Ekiert
}

Received: 27 December 2012/Accepted: 12 June 2013/Published online: 27 June 2013

(C) The Author(s) 2013. This article is published with open access at Springerlink.com

\begin{abstract}
Phenolic acids, both benzoic and cinnamic acid derivatives, are plant metabolites with high therapeutic and cosmetic values. Methanolic extracts from the biomass of shoot and callus cultures of Aronia melanocarpa growing on seven variants of the Murashige and Skoog (MS) medium with different concentrations of plant growth regulators, BA and NAA, ranging from 0.1 to $3.0 \mathrm{mg} \mathrm{l}^{-1}$, were examined for the production of free phenolic acids and cinnamic acid using the high-performance liquid chromatography (HPLC) method. The extracts from the shoot and callus cultures were confirmed to contain five of the twelve compounds tested for: caffeic, p-coumaric, p-hydroxybenzoic, syringic and vanillic acids. The shoot extracts contained additionally salicylic acid. Both the total amounts and the amounts of individual compounds in either the shoot or callus extracts were dependent on the concentration of cytokinin and auxin in the MS medium variants. The total amounts in the shoot and callus cultures were in the range from 93.52 to $217.00 \mathrm{mg}$ $100 \mathrm{~g}^{-1} \mathrm{DW}$ and from 47.11 to $83.83 \mathrm{mg} 100 \mathrm{~g}^{-1} \mathrm{DW}$, respectively. The amounts of individual compounds showed wide variation, from 1.31 to $91.86 \mathrm{mg} 100 \mathrm{~g}^{-1} \mathrm{DW}$ in the

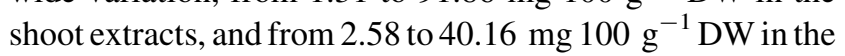

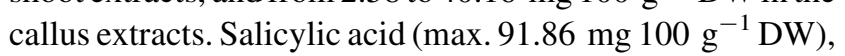
p-coumaric acid (max. $\left.62.39 \mathrm{mg} 100 \mathrm{~g}^{-1} \mathrm{DW}\right)$ and p-hydroxybenzoic acid (max. $50.66 \mathrm{mg} 100 \mathrm{~g}^{-1} \mathrm{DW}$ ) dominated in the shoot extracts, while syringic acid (max. $40.16 \mathrm{mg}$ $100 \mathrm{~g}^{-1} \mathrm{DW}$ ) and p-hydroxybenzoic acid (max. $23.59 \mathrm{mg}$ $100 \mathrm{~g}^{-1} \mathrm{DW}$ ) were the main metabolites in the callus
\end{abstract}

\footnotetext{
A. Szopa $(\square) \cdot$ H. Ekiert

Department of Pharmaceutical Botany, Collegium Medicum, Jagiellonian University, ul. Medyczna 9, 30-688 Kraków, Poland e-mail: a.szopa@uj.edu.pl

H. Ekiert

e-mail: mfekiert@cyf-kr.edu.pl
}

extracts. This is the first report on the quantitative analysis of benzoic and cinnamic acid derivatives in shoot and callus cultures of A. melanocarpa growing on MS-based media with different concentrations of selected plant growth regulators-BA and NAA. The obtained maximum amounts of some metabolites are of interest from a practical perspective.

Keywords Rosaceae $\cdot$ Black chokeberry $\cdot$ Shoot culture $\cdot$ Callus culture $\cdot$ Plant growth regulators $\cdot$ Free phenolic acids
Abbreviations
BA 6-Benzyladenine
DW Dry weight
HPLC High-performance liquid chromatography
LS Linsmaier and Skoog
NAA $\alpha$-Naphthaleneacetic acid
MS Murashige and Skoog

\section{Introduction}

Phenolic acids, derivatives of benzoic and cinnamic acids, and depsides, e.g. chlorogenic and rosmarinic acids, are a therapeutically important group of plant metabolites. These compounds possess long-known anti-inflammatory, cholagogic, spasmolytic, hypolipemic and antiaggregatory properties (De Graff et al. 2003; Ekiert and Czygan 2007; Nakamura et al. 2001; Sroka and Cisowski 2003). More recent studies have also revealed significant antioxidant, anti-free radical, immunostimulating and anticancer activities of some representatives of this group, e.g. protocatechuic acid, caffeic acid and chlorogenic acid (Chlopčikova et al. 2004; Jiang et al. 2000; Kono et al. 
1995; Olthof et al. 2001). Hydroxybenzoic acids possess many other very important biological properties. pHydroxybenzoic acid exhibits antimicrobial, antifungal, antisickling and estrogenic activities (Chong et al. 2009; Pugazhendhi et al. 2005). o-Hydroxybenzoic acid (salicylic acid) has antiinflammatory, antiseptic, antifungal, antipyretic, analgesic and keratolitic properties (Lin and Nakatsui 1998). Vanillic acid (monomethoxy-derivative of phydroxybenzoic acid) exhibits antisickling and anthelmintic, as well as hepatoprotective, activities (Itoh et al. 2009, 2010). Syringic acid (dimethoxy-derivative of p-hydroxybenzoic acid), besides being an antioxidant, shows antibacterial and hepatoprotective activities (Itoh et al. 2009, 2010; Kong et al. 2008). Several publications have emphasized valuable therapeutic and also cosmetic properties of p-coumaric acid, e.g. its antioxidant and antigenotoxic effects, and its potential usefulness as a hypopigmenting agent (Abdel-Wahab et al. 2003; An et al. 2010; Ferguson et al. 2005).

Aronia melanocarpa (Rosaceae) is a medicinal, cosmetic and culinary plant species, native to North America, cultivated in Europe. The fruits of this species are a rich source of different groups of metabolites, e.g. flavonoids, anthocyanins, tannins, pectins, vitamin $\mathrm{C}$ and bioelements. Of phenolic acids, the presence of depsides: chlorogenic acid and its isomer, isochlorogenic acid, has been confirmed (Kokotkiewicz et al. 2010; Kulling and Rawel 2008; Oszmański and Wojdyło 2005; Silmestad et al. 2005; Zdunczyka et al. 2002). Other compounds of this group of metabolites were documented for the first time by our team to occur in fruit extracts of soil-grown plants; they included caffeic, p-coumaric, p-hydroxybenzoic, syringic, vanillic and salicylic acids (Szopa et al. 2013). However, our studies did not confirm the presence of chlorogenic acid, which had been reported earlier by Kulling and Rawel (2008).

The capacity of plant in vitro cultures to accumulate phenolic acids is an interesting scientific phenomenon in plant biotechnology. There are some examples of successful production of, for instance, rosmarinic acid in in vitro cultures of many Lamiaceae and Boraginaceae species (Ekiert et al. 2013; Makri and Kintzios 2004), ellagic acid in shoot cultures of Rubus chamaemorus (Thiem and Krawczyk 2003), protocatechuic acid in shoot cultures of Ruta graveolens (Ekiert et al. 2008, 2009) and p-coumaric acid in shoot-differentiating callus cultures of Ruta graveolens ssp. divaricata (Ekiert et al. 2008; Piekoszewska et al. 2008). There has also been a report recently on a high production of two depsides, rosmarinic and chlorogenic acids, in in vitro cultures of Eringium planum (Kikowska et al. 2012).

Biotechnological studies carried out in our laboratory have demonstrated the ability of in vitro cultures of
A. melanocarpa at different stages of differentiation (shoot and callus cultures) to accumulate free phenolic acids on some variants of the Linsmaier and Skoog (LS, 1965) medium with different concentrations of plant growth regulators, BA and NAA. The obtained results, namely high maximum amounts of some hydroxybenzoic acids, e.g. p-hydroxybenzoic and salicylic (o-hydroxybenzoic) acids, and other biologically active phenolic acids indicated that the study on the accumulation of this group of metabolites should be continued. Our studies have documented that in vitro cultures of A. melanocarpa can be a good model for examining the accumulation of phenolic acids (Szopa et al. 2013).

The aim of the present study was to investigate the accumulation of free phenolic acids in the biomass from $A$. melanocarpa shoot and callus cultures maintained on seven variants of the Murashige and Skoog medium (1962) containing different amounts of growth regulators, BA and NAA (which ranged from 0.1 to $3 \mathrm{mg} \mathrm{l}^{-1}$ of medium). Eleven free phenolic acids and cinnamic acid, a biogenetic precursor of one of the subgroups of these compounds, were determined in methanolic extracts from the obtained biomass with the HPLC method. Our expectation was that by optimizing the composition of the culture media, we would increase even more the production of biologically active metabolites.

\section{Materials and methods}

\section{Establishment of in vitro cultures}

The in vitro cultures (shoot and callus cultures) of $A$. melanocarpa (Michx.) Elliott were established from leaf buds of plants growing in Rogów Arboretum, Warsaw University of Life Sciences, Forest Experimental Station in Rogów, Poland (for details see Szopa et al. 2013). In short, initiation of undifferentiating callus tissue was observed on some bud fragments, while from some others microshoots emerged. After 4 weeks, two different cultures were obtained—-shoot cultures and callus cultures.

\section{Experimental in vitro cultures}

Experimental in vitro cultures of both types-shoot and callus cultures, were maintained on seven variants of the Murashige and Skoog (MS 1962) solid medium with different concentrations of plant growth regulators, BA and NAA $\left(\mathrm{mg} \mathrm{l}^{-1}\right)$ : 0.1 and $2.0 ; 0.5$ and $2.0 ; 1.0$ and $0.5 ; 2.0$ and $0.5 ; 2.0$ and $1.0 ; 2.0$ and $2.0 ; 3.0$ and 1.0. The inoculum was 1 gram for shoot cultures, and 2 grams for callus cultures. The cultures (three series) were grown under 
constant artificial light $\left(4 \mathrm{~W} \mathrm{~m}{ }^{-2}\right.$, LF-40 W lamp, daylight, Piła) at $25 \pm 2{ }^{\circ} \mathrm{C}$ for four weeks.

\section{Extraction and HPLC analysis}

The dried biomass from in vitro cultures collected after 4-week growth cycles and plant material (0.5 g) were extracted twice with boiling methanol for $3 \mathrm{~h}$. Eleven phenolic acids and cinnamic acid were quantified in methanolic extracts using the HPLC method developed by Tian et al. (2005). Separation was performed using a Kinetex $^{\mathrm{TM}} \mathrm{C} 18$ analytical column $(150 \times 4.6 \mathrm{~mm}, 2.6 \mu \mathrm{m})$ at $25{ }^{\circ} \mathrm{C}$. The mobile phase consisted of $0.1 \%$ trifluoroacetic acid (A) and acetonitrile (B), and the flow rate was $1.0 \mathrm{ml} \mathrm{min} .^{-1}$ (gradient program); injection volume was $5 \mu$. Detection wavelength was set at $254 \mathrm{~nm}$. Quantification was made by comparison with standard solutions (from 0.015625 to $0.25 \mathrm{mg} \mathrm{ml}^{-1}$ ). Retention times $-t_{R}$ (min.) of phenolic acids and cinnamic acid standards were as follows: protocatechuic-4.7, chlorogenic-5.5, phydroxybenzoic - 5.9, caffeic - 6.9, syringic - 7.2, vanillic 8.2, sinapic-12.8, ferulic-13.1, p-coumaric-14.3, salicylic-19.6, rosmarinic_-26.0, cinnamic_-26.5 (Sigma and Fluka).

\section{Results}

Shoot cultures

In the experimental $A$. melanocarpa shoot cultures maintained on seven variants of MS medium, the increases in dry biomass over 4-week growth cycles were very high, from 11.4- to 17.2-times. A more than 15-fold increase in dry shoot biomass was observed on four of the seven MS medium variants, namely on those that contained BA and NAA at the following concentrations: 2 and $2 \mathrm{mg} \mathrm{l}^{-1}, 0.1$ and $2 \mathrm{mg} \mathrm{l}^{-1}$, 0.5 and $2 \mathrm{mg} \mathrm{l}^{-1}, 3$ and $1 \mathrm{mg} \mathrm{l}^{-1}$, respectively. The greatest biomass growth was seen on the medium supplemented with auxin and cytokinin at $2 \mathrm{mg} \mathrm{l}^{-1}$ each.

Methanolic extracts from the biomass cultured on the tested MS medium variants contained six of the twelve compounds under analysis: caffeic, p-coumaric, p-hydroxybenzoic, salicylic, syringic and vanillic acids. None of the extracts analyzed contained: chlorogenic, cinnamic, ferulic, protocatechuic, rosmarinic or sinapic acids (Fig. 1).

The total amounts of the compounds under analysis varied widely, ranging from 93.52 to $217.50 \mathrm{mg} 100 \mathrm{~g}^{-1} \mathrm{DW}$. High

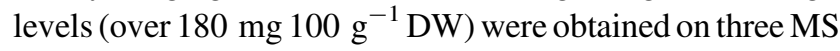
medium variants containing the following concentrations of

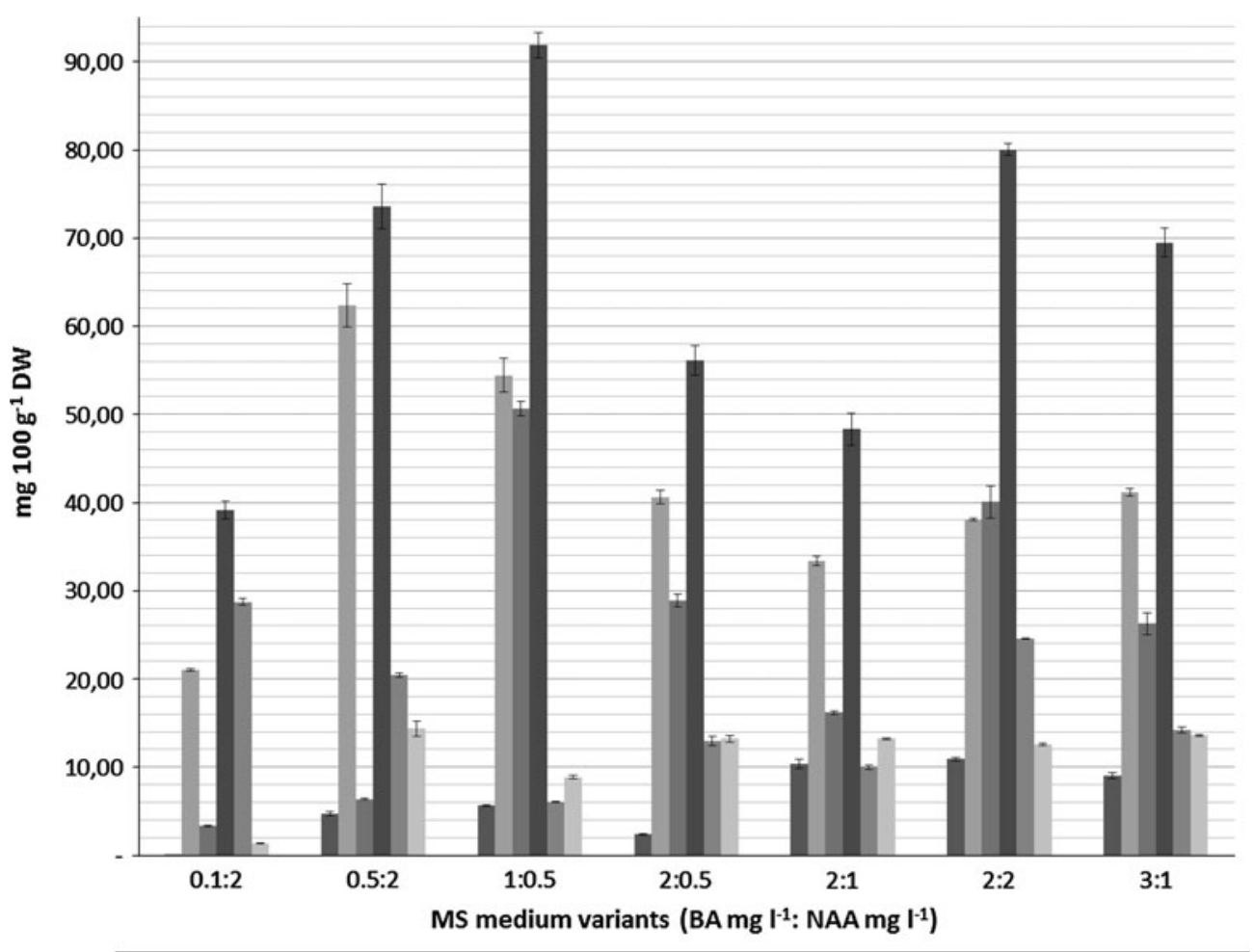

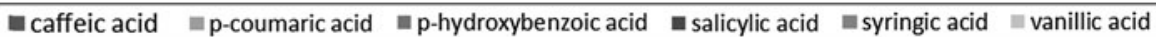

Fig. 1 Contents (mg $100 \mathrm{~g}^{-1} \mathrm{DW}$ ) of phenolic acids in biomass extracts from shoot cultures of A. melanocarpa cultivated on MS medium variants with different concentrations of BA and NAA. The values are means of three experiments \pm SD 
BA and NAA: 1 and $0.5 \mathrm{mg} \mathrm{l}^{-1}, 2$ and $2 \mathrm{mg} \mathrm{l}^{-1}$, and 0.5 and $2 \mathrm{mg} \mathrm{l}^{-1}$. The maximum amounts were found on the medium containing $1 \mathrm{mg} \mathrm{l}^{-1} \mathrm{BA}$ and $0.5 \mathrm{mg}^{-1} \mathrm{NAA}$. The amounts of individual compounds also varied widely, ranging from hundredths of a milligram to almost $92 \mathrm{mg} 100 \mathrm{~g}^{-1} \mathrm{DW}$, and their medium-dependent amounts differed from 2.5- to 181.7times. The maximum amounts of two metabolites, caffeic acid and vanillic acid, were of a similar order of magnitude: 11-14 mg $100 \mathrm{~g}^{-1} \mathrm{DW}$ (Table 1). The caffeic acid content exceeded $9 \mathrm{mg} 100 \mathrm{~g}^{-1} \mathrm{DW}$ on three variants of MS medium. Accumulation of this compound was especially stimulated on the medium enriched with cytokinin and auxin at $2 \mathrm{mg} \mathrm{l}^{-1}$ each (max. $10.9 \mathrm{mg} 100 \mathrm{~g}^{-1} \mathrm{DW}$ ). The amounts of vanillic acid on as many as four MS medium variants were higher than $13 \mathrm{mg} 100 \mathrm{~g}^{-1} \mathrm{DW}$. The maximum amount of this compound (14.36 mg $100 \mathrm{~g}^{-1} \mathrm{DW}$ ) was noted on the medium supplemented with $0.5 \mathrm{mg}^{-1} \mathrm{BA}$ and $2.0 \mathrm{mg} \mathrm{l}^{-1} \mathrm{NAA}$. The maximum amounts of syringic acid were markedly higher, reaching about $30 \mathrm{mg} 100 \mathrm{~g}^{-1} \mathrm{DW}$, with the maximum content of $28.72 \mathrm{mg} 100 \mathrm{~g}^{-1} \mathrm{DW}$ on the medium containing $0.1 \mathrm{mg} \mathrm{l^{-1 }}$ BA and $2 \mathrm{mg} 1^{-1}$ NAA. High amounts of it (more than $20 \mathrm{mg} 100 \mathrm{~g}^{-1} \mathrm{DW}$ ) were also obtained on two other MS medium variants $\left(0.5 \mathrm{mg} \mathrm{l}^{-1} \mathrm{BA}\right.$ and $2 \mathrm{mg} \mathrm{l}^{-1} \mathrm{NAA}$, and $2 \mathrm{mg}^{-1} \mathrm{BA}$ and $2 \mathrm{mg} \mathrm{l}^{-1} \mathrm{NAA}$ ). The amounts of the other three phenolic acids were of interest from a practical point of view, equal to $50.66 \mathrm{mg} 100 \mathrm{~g}^{-1} \mathrm{DW}$ for $\mathrm{p}$-hydroxybenzoic acid, $62.39 \mathrm{mg} 100 \mathrm{~g}^{-1} \mathrm{DW}$ for $\mathrm{p}$-coumaric acid, and $91.86 \mathrm{mg} 100 \mathrm{~g}^{-1} \mathrm{DW}$ for salicylic acid.

The maximum amounts of p-hydroxybenzoic acid and salicylic acid were obtained on the media containing $1 \mathrm{mg}^{-1} \mathrm{BA}$ and $0.5 \mathrm{mg}^{-1}$ NAA. High amounts of these metabolites, equal to 40.06 and $80.05 \mathrm{mg} 100 \mathrm{~g}^{-1} \mathrm{DW}$, respectively, were confirmed in the extracts from shoots cultivated on the MS medium containing $2 \mathrm{mg} \mathrm{l}^{-1}$ cytokinin and $2 \mathrm{mg} \mathrm{l}^{-1}$ auxin. The highest quantities of p-coumaric acid were obtained on the MS medium supplemented with $0.5 \mathrm{mg} \mathrm{l}^{-1} \mathrm{BA}$ and $2 \mathrm{mg} \mathrm{l}^{-1} \mathrm{NAA}$

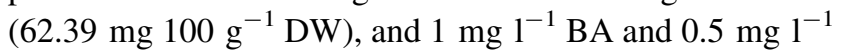
NAA (54.71 mg $\left.100 \mathrm{~g}^{-1} \mathrm{DW}\right)$.
Callus cultures

The increases in the biomass of callus cultures over 4-week growth cycles were relatively low (3.3- to 5.0-fold). The highest biomass growth was observed on two MS medium variants containing $2 \mathrm{mg} \mathrm{l}^{-1} \mathrm{BA}$ and $2 \mathrm{mg}^{-1} \mathrm{NAA}$, and $0.5 \mathrm{mg} \mathrm{l}^{-1} \mathrm{BA}$ and $2 \mathrm{mg}^{-1} \mathrm{NAA}$ (a 5.0- and 4.9-fold increase, respectively).

Extracts of the biomass from A. melanocarpa callus cultures contained five of the twelve compounds under study: caffeic, p-coumaric, p-hydroxybenzoic, syringic and vanillic acids, i.e. the same as those found in the shoot culture extracts (Fig. 2). However, undifferentiating callus did not contain salicylic acid dominating in the shoots. Chlorogenic, cinnamic, protocatechuic, rosmarinic, sinapic and ferulic acids were also not identified in any of the extracts.

The total amounts of five metabolites varied from about 47 to $84 \mathrm{mg} 100 \mathrm{~g}^{-1} \mathrm{DW}$ depending on the concentrations of the growth regulators in the media. The highest total concentration of phenolic acids was obtained in the biomass growing on the MS medium supplemented with BA and NAA at different concentrations: 2 and $1 \mathrm{mg} \mathrm{l}^{-1} ; 0.5$ and $2 \mathrm{mg}^{-1}$, and 1 and $0.5 \mathrm{mg} \mathrm{l}^{-1}$, respectively. The amounts of individual compounds were within a wide range, from about 2.6 to $40 \mathrm{mg} 100 \mathrm{~g}^{-1} \mathrm{DW}$, depending on the MS medium variant. The amounts of individual phenolic acids differed widely from 1.8- to 4.0-times, depending on the MS medium variant. Two metabolites dominated: syringic acid (max. $40.16 \mathrm{mg} 100 \mathrm{~g}^{-1} \mathrm{DW}$ )

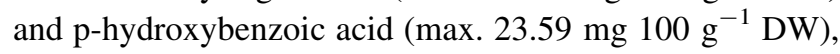
whereas the amounts of the remaining three phenolic acids: caffeic, p-coumaric and vanillic acids, did not exceed $11 \mathrm{mg} 100 \mathrm{~g}^{-1}$ DW (Table 1).

The highest syringic acid content was found to occur in the extracts of callus growing on the MS medium supplemented with $2 \mathrm{mg} \mathrm{l}^{-1} \mathrm{BA}$ and $1 \mathrm{mg} \mathrm{l}^{-1} \mathrm{NAA}$, and $1 \mathrm{mg}^{-1}$ BA and $0.5 \mathrm{mg} \mathrm{l}^{-1} \mathrm{NAA}$ (40.16 and $38.96 \mathrm{mg}$ $100 \mathrm{~g}^{-1} \mathrm{DW}$, respectively). Extracts of the biomass cultivated on five MS medium variants contained high

Table 1 Contents $\left(\mathrm{mg} 100 \mathrm{~g}^{-1} \mathrm{DW} \pm \mathrm{SD}\right)$ of free phenolic acids in fruit extracts of soil-grown plants and their maximal contents in biomass extracts from in vitro cultures

\begin{tabular}{lccccccc}
\hline Plant material & \multicolumn{2}{l}{ Phenolic acids* } \\
\cline { 2 - 8 } & Caffeic acid & p-Coumaric acid & p-Hydroxy-benzoic acid & Salicylic acid & Syringic acid & Vanillic acid & Total contents \\
\hline Shoot cultures & $10.90 \pm 0.17$ & $62.39 \pm 2.44$ & $50.66 \pm 0.84$ & $91.86 \pm 1.44$ & $28.72 \pm 0.41$ & $14.36 \pm 0.84$ & $217.50 \pm 7.09$ \\
Callus cultures & $8.08 \pm 1.12$ & $10.93 \pm 0.40$ & $23.59 \pm 0.33$ & nd & $40.16 \pm 1.13$ & $7.47 \pm 0.07$ & $83.84 \pm 3.05$ \\
Fruits** & $3.96 \pm 0.06$ & $3.05 \pm 0.07$ & $5.29 \pm 0.04$ & $15.60 \pm 0.18$ & $4.16 \pm 0.06$ & $0.37 \pm 0.02$ & $32.43 \pm 0.43$ \\
\hline
\end{tabular}

* Maximal contents obtained on different MS medium variants

** Our earlier results (Szopa et al. 2013)

"nd"-not detected in analyzed biomass extracts 


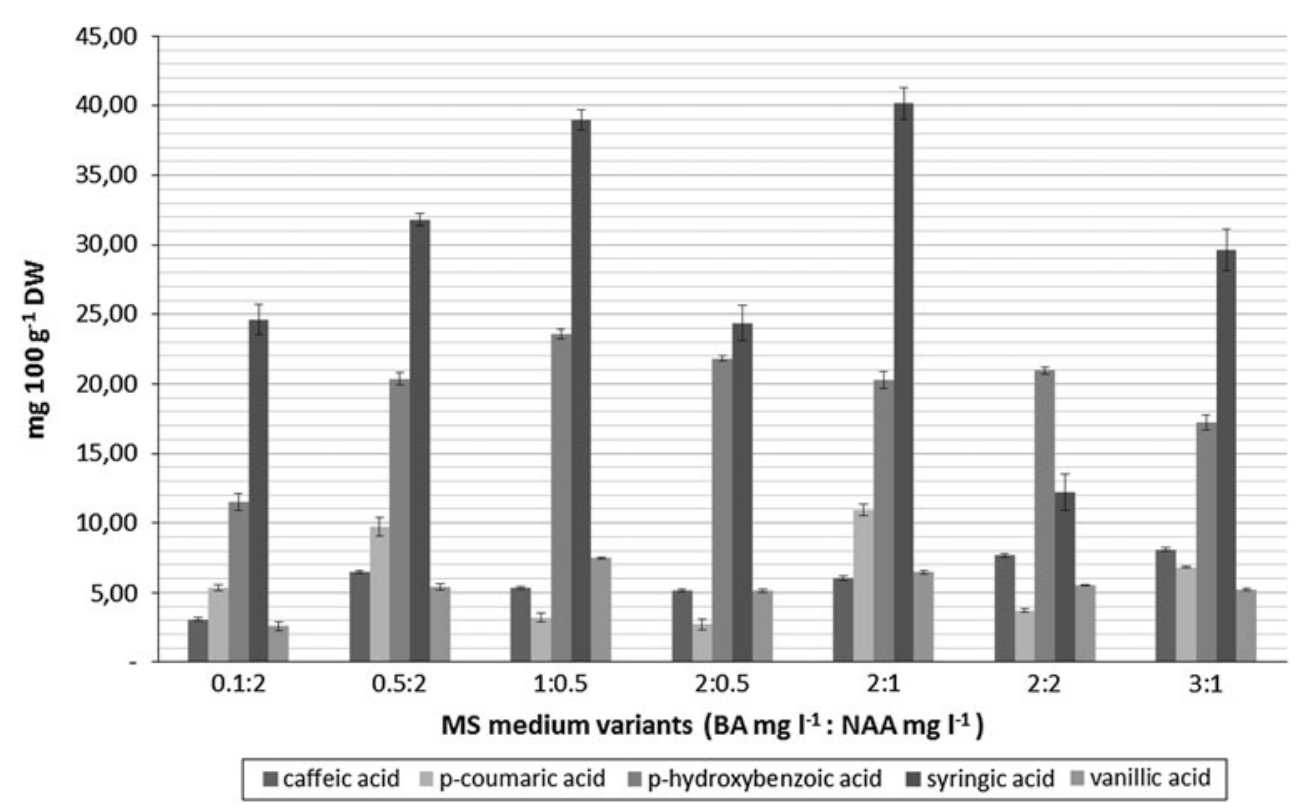

Fig. 2 Contents (mg $100 \mathrm{~g}^{-1} \mathrm{DW}$ ) of phenolic acids in biomass extracts from callus cultures of A. melanocarpa cultivated on MS medium variants with different concentrations of BA and NAA. The values are means of three experiments \pm SD

amounts of p-hydroxybenzoic acid (over $20 \mathrm{mg} 100 \mathrm{~g}^{-1}$ DW). Maximum amounts were obtained on MS variants containing $2 \mathrm{mg} \mathrm{l}^{-1} \mathrm{BA}$ and $1 \mathrm{mg} \mathrm{l}^{-1} \mathrm{NAA}$. The maximum amount of caffeic acid $\left(8.08 \mathrm{mg} 100 \mathrm{~g}^{-1} \mathrm{DW}\right)$ was documented on the medium enriched with $3 \mathrm{mg}^{-1} \mathrm{BA}$ and $1 \mathrm{mg}^{-1} \mathrm{NAA}$, while the highest amount of p-coumaric acid (10.93 mg $\left.100 \mathrm{~g}^{-1} \mathrm{DW}\right)$ was on the medium containing $2 \mathrm{mg} \mathrm{l}^{-1} \mathrm{BA}$ and $1 \mathrm{mg} \mathrm{l}^{-1}$ NAA. The vanillic acid content on six of the seven MS medium variants tested exceeded $5 \mathrm{mg} 100 \mathrm{~g}^{-1} \mathrm{DW}$. This metabolite accumulated in the greatest amounts on the medium containing $1 \mathrm{mg} \mathrm{l}^{-1}$ BA and $0.5 \mathrm{mg} \mathrm{l}^{-1} \mathrm{NAA}$ (max. $7.47 \mathrm{mg} 100 \mathrm{~g}^{-1} \mathrm{DW}$ ).

\section{Discussion}

Both the total amounts of free phenolic acids under study and the amounts of individual compounds in the two types of in vitro cultures of A. melanocarpa differing in the degree of differentiation, namely shoot and callus cultures, varied widely depending on BA and NAA concentrations and the cytokinin/auxin ratio in the tested variants of the Murashige and Skoog (MS, 1962) medium.

In the shoot cultures, the total amounts of free phenolic acids increased up to 2.33-times, while the amounts of individual compounds varied from 2.5- to 181.7-times depending on the MS medium variant. In the undifferentiating callus cultures, the total amounts of free phenolic acids also varied, and increased up to 1.78-times. The amounts of individual metabolites differed widely from 1.8- to 4.0-times. In shoot extracts, the pattern of the phenolic acids being determined was similar for MS media with a 2:1, 3:1 and 4:1 cytokinin/auxin ratio. The same situation was observed on MS media with a 1:4 and 1:20 cytokinin/auxin ratio. In callus extracts, the relationship was the same. On MS media with a 2:1, 3:1 and 4:1 cytokinin/auxin ratio, the patterns of the compounds being determined were similar. Likewise, on MS media with a 1:4 and 1:20 cytokinin/auxin ratio the pattern of the estimated phenolic acids was also similar.

The effect of plant growth regulators in culture media, i.e. their concentration and the cytokinin/auxin ratio, on the accumulation of different groups of secondary metabolites is a well-known phenomenon (Ramawat and Mathur 2007). We documented this relationship in our earlier studies on the accumulation of other groups of secondary metabolites in in vitro cultures, e.g. linear furanocoumarins in Ammi majus (Ekiert 1993; Ekiert and Gomólka 2000a) and Pastinaca sativa (Ekiert and Gomólka 2000b) cultures, and on lignan accumulation in Schisandra chinensis cultures (Szopa and Ekiert 2011, 2013). We also observed the dependence of free phenolic acid accumulation on the composition of growth regulators in Ruta graveolens shoot cultures (Ekiert et al. 2009) and in Ruta graveolens ssp. divaricata shoot-differentiating callus cultures (Ekiert et al. 2008; Piekoszewska et al. 2008), as well as in S. chinensis shoot-differentiating callus and undifferentiating callus cultures (Szopa and Ekiert 2012). In our earlier studies, we demonstrated empirically the significance of the concentration of plant growth regulators and the cytokinin/auxin ratio in the media for the accumulation of free phenolic acids in A. melanocarpa shoot and callus cultures maintained on several variants of 
Linsmaier and Skoog (LS, 1965) medium with different BA and NAA concentrations (Szopa et al. 2013).

High total amounts of free phenolic acids (over $180 \mathrm{mg}$ $100 \mathrm{~g}^{-1} \mathrm{DW}$ ) in the shoot cultures of A. melanocarpa were obtained on three MS media containing $1 \mathrm{mg}^{-1}$ BA and $0.5 \mathrm{mg}^{-1} \mathrm{NAA}, 2 \mathrm{mg}^{-1} \mathrm{BA}$ and $2 \mathrm{mg}^{-1} \mathrm{NAA}$, and $0.5 \mathrm{mg} \mathrm{l}^{-1} \mathrm{BA}$ and $2 \mathrm{mg}^{-1} \mathrm{NAA}$. On two of these variants, namely those containing $2 \mathrm{mg}^{-1} \mathrm{BA}$ and $2 \mathrm{mg}^{-1} \mathrm{NAA}$, and $0.5 \mathrm{mg}^{-1} \mathrm{BA}$ and $2 \mathrm{mg}^{-1} \mathrm{NAA}$, high increases in biomass were observed. Therefore, these two variants of the MS medium can be proposed as universal, both "productive" and "growth-promoting", media for shoot culture.

Relatively high amounts of phenolic acids (over $70 \mathrm{mg}$ $100 \mathrm{~g}^{-1} \mathrm{DW}$ ) were found in the extracts from the biomass of callus cultures grown on MS media containing $2 \mathrm{mg} \mathrm{l}^{-1}$ BA and $1 \mathrm{mg} \mathrm{l}^{-1} \mathrm{NAA}, 0.5 \mathrm{mg}^{-1} \mathrm{BA}$ and $2 \mathrm{mg} \mathrm{l}^{-1}$ $\mathrm{NAA}$, and $1 \mathrm{mg} \mathrm{l}^{-1} \mathrm{BA}$ and $0.5 \mathrm{mg} \mathrm{l}^{-1} \mathrm{NAA}$. On one of these variants, the one containing $0.5 \mathrm{mg} \mathrm{l}^{-1} \mathrm{BA}$ and $2 \mathrm{mg}^{-1}$ NAA, the increases in dry biomass were among the highest. This MS variant can be proposed as a universal, both "productive" and "growth-promoting", medium for callus culture.

Shoot cultures of A. melanocarpa had a higher capacity to accumulate phenolic acids than its callus cultures. The obtained total amounts of metabolites in shoot extracts were from 1.57- to 4.12-times higher than those obtained in the extracts from undifferentiating-callus cultures maintained on identical variants of MS medium.

Such a relationship is known in plant biotechnology (Charlwood et al. 1990). It was also shown in our studies on lignans accumulation in $S$. chinensis cultures at different stages of differentiation (Szopa and Ekiert 2011, 2013) and in Ruta graveolens and Ruta graveolens ssp. divaricata cultures, which accumulated different amounts of linear furanocoumarins depending on the degree of organogenesis (Ekiert and Czygan 2007). Likewise, shoots from $R$. graveolens in vitro cultures (Ekiert et al. 2009) and shootdifferentiating callus cultures from Ruta graveolens ssp. divaricata contained considerable amounts of phenolic acids (Ekiert et al. 2008; Piekoszewska et al. 2008). In the case of our earlier studies on the accumulation of free phenolic acids in in vitro cultures of Schisandra chinensis, we had obtained a surprising result (Szopa and Ekiert 2012). The total amounts of phenolic acids determined in the extracts from undifferentiating-callus cultures were greater than those in the extracts from shoot-differentiating callus cultures.

In our earlier studies with A. melanocarpa cultivated on variants of the LS medium, we had observed a more typical relationship. The maximum total amount of phenolic acids in the biomass of A. melanocarpa shoot cultures was 2.6times higher than in the biomass of undifferentiating callus cultures (Szopa et al. 2013).

The obtained results indicated that in the shoot cultures the biosynthetic pathways of benzoic acid derivatives concurred with those of cinnamic acid derivatives. p-Hydroxybenzoic acid and salicylic acid (o-hydroxybenzoic acid), derivatives of benzoic acid, were the main metabolites; however, p-coumaric acid, a derivative of cinnamic acid, was also accumulated in notable amounts. The results obtained with the callus cultures of A. melanocarpa indicated that the predominant biosynthetic pathways were those of the benzoic acid derivatives. Syringic acid (dimetoxy-derivative of benzoic acid) and p-hydroxybenzoic acid were the metabolites accumulated in the greatest quantities.

The amounts of some phenolic acids in the biomass from the shoot and callus cultures were interesting from a practical perspective.

Salicylic acid, p-hydroxybenzoic acid and p-coumaric acid were accumulated in shoots in marked quantities (max. content about 90, 60 and $50 \mathrm{mg} 100 \mathrm{~g}^{-1} \mathrm{DW}$, respectively). In the callus cultures, two compounds were accumulated in quantities that are of interest from a practical point of view, namely: syringic acid (max. about $40 \mathrm{mg} 100 \mathrm{~g}^{-1} \mathrm{DW}$ ) and p-hydroxybenzoic acid (max.

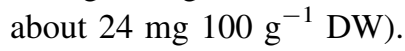

In our earlier studies on A. melanocarpa in vitro cultures, we had analyzed fruit extracts of soil-grown plants.

Table 2 Comparison of amounts $\left(\mathrm{mg} 100 \mathrm{~g}^{-1} \mathrm{DW} \pm \mathrm{SD}\right)$ of selected free phenolic acids obtained in biomass extracts from shoot cultures of A. melanocarpa cultivated on MS and LS media variants with identical concentrations of BA and NAA

\begin{tabular}{|c|c|c|c|c|c|c|c|c|c|}
\hline \multicolumn{2}{|c|}{ Media variants } & \multicolumn{2}{|c|}{ p-Hydroxybenzoic acid } & \multicolumn{2}{|l|}{ Salicylic acid } & \multicolumn{2}{|c|}{ p-Coumaric acid } & \multicolumn{2}{|l|}{ Total contents } \\
\hline $\begin{array}{l}\text { BA:NAA } \\
\text { ratio }\end{array}$ & $\begin{array}{l}\text { BA:NAA } \\
\left(\mathrm{mg}^{-1}\right)\end{array}$ & MS & LS* & MS & LS & MS & LS & MS & LS \\
\hline $2: 1$ & $1: 0.5$ & $50.66 \pm 0.84$ & $38.73 \pm 0.53$ & $91.86 \pm 1.45$ & $51.64 \pm 1.67$ & $54.44 \pm 1.88$ & nd & $217.50 \pm 7.09$ & $119.41 \pm 3.36$ \\
\hline $1: 1$ & $2: 2$ & $40.06 \pm 1.79$ & $27.18 \pm 0.77$ & $80.05 \pm 0.69$ & $49.79 \pm 4.43$ & $38,08 \pm 0.16$ & nd & $206.19 \pm 3.01$ & $103.05 \pm 6.16$ \\
\hline $3: 1$ & $3: 1$ & $26.25 \pm 1.24$ & $18.35 \pm 0.28$ & $69.47 \pm 1.62$ & $77.52 \pm 1.01$ & $41.15 \pm 0.47$ & nd & $173.72 \pm 4.07$ & $109.25 \pm 1.65$ \\
\hline
\end{tabular}

* Our earlier results (Szopa et al. 2013)

"nd"-not detected in the analyzed biomass extracts 
The extracts were not shown to contain chlorogenic acid, which is considered to be a metabolite characteristic for this species. For the first time, we had confirmed the presence of six other phenolic acids. The main compounds were salicylic acid and p-hydroxybenzoic acid. The other four: caffeic, p-coumaric, syringic and vanillic acids were found to be present in lower quantities, below $4.2 \mathrm{mg}$ $100 \mathrm{~g}^{-1}$ DW (Table 1).

The maximum total amounts of phenolic acids obtained in in vitro cultures on the tested variants of MS medium were about 7-times (shoot culture) and 2.5-times (callus culture) higher than in the fruit extracts $\left(32.43 \mathrm{mg} 100 \mathrm{~g}^{-1}\right.$ DW).

Comparison of the total amounts of the metabolites analyzed for in shoot cultures maintained on MS and LS media supplemented with identical concentrations of growth regulators, BA and NAA (three media variants), indicates that 1.6- to 2.0-times higher amounts of phenolic acids can be obtained on the MS variants (Table 2). Some metabolites, namely salicylic acid, p-hydroxybenzoic acid and syringic acid were also accumulated on the MS medium variants in amounts from 1.1- to 1.8-times greater. Moreover, p-coumaric acid was confirmed on those media. It is likely that the richer chemical composition of the Murashige and Skoog medium (1962), e.g. vitamins, stimulate different enzymatic reactions in the biomass of in vitro cultured shoots.

The total amounts of the phenolic acids determined in undifferentiating-callus cultures cultivated on two identical variants of MS and LS media were almost the same. Only on one variant, containing $3 \mathrm{mg}^{-1} \mathrm{BA}$ and $1 \mathrm{mg} \mathrm{l}^{-1}$ NAA, the total content was 1.22-times higher on the MSbased than LS-based medium. On the other hand, in undifferentiating-callus cultures of Anethum graveolens established in our laboratory and maintained on MS and LS media with $\mathrm{BA}$ and $\mathrm{NAA}$ at $2 \mathrm{mg} \mathrm{l}^{-1}$ each, 2.5-times higher total amounts of phenolic acids and 1.2-5.5-times higher amounts of individual phenolic acids (vanillic, p-hydroxybenzoic and salicylic acids) were obtained on MS-based media. The accumulation of two linear furanocoumarins: bergapten and imperatorin, was also higher, 1.94- and 1.84-times, respectively, in those A. graveolens callus cultures (submitted for publication).

The in vitro cultures of A. melanocarpa cultivated on the different variants of MS medium produced notable amounts of some phenolic acids, compounds with high therapeutic and cosmetic values. We propose shoot cultures of A. melanocarpa as a rich biotechnological source of salicylic, p-coumaric and p-hydroxybenzoic acids, and its callus cultures as a potential source of syringic acid.

Based on the present studies, we can also propose the MS medium supplemented with $0.5 \mathrm{mg} \mathrm{l}^{-1} \mathrm{BA}$ and $2 \mathrm{mg} \mathrm{l}^{-1}$ $\mathrm{NAA}$, and $2 \mathrm{mg}^{-1} \mathrm{BA}$ and $2 \mathrm{mg} \mathrm{l}^{-1} \mathrm{NAA}$ as the best media for A. melanocarpa shoot cultures in terms of biomass growth and accumulation of phenolic acids, and the MS medium supplemented with $0.5 \mathrm{mg} \mathrm{l}^{-1} \mathrm{BA}$ and $2 \mathrm{mg}^{-1} \mathrm{NAA}$ as the best "universal" medium for callus culture.

Acknowledgments The authors wish to express their sincere gratitude to Mr P. Banaszczak and Mr J. Szkup from the Rogów Arboretum-Warsaw University of Life Sciences, Forest Experimental Station in Rogów (Poland) for plant material. The authors wish to express their sincere gratitude, also to Dr. R. Wróbel for translating this article into English and to Dr. M. Paszt for professional English correction.

Open Access This article is distributed under the terms of the Creative Commons Attribution License which permits any use, distribution, and reproduction in any medium, provided the original author(s) and the source are credited.

\section{References}

Abdel-Wahab MH, El-Mahdy MA, Abd-Ellah MF, Helal GK, Khalifa F, Hamada FM (2003) Influence of p-coumaric acid on doxorubicin-induced oxidative stress in rat's heart. Pharmacol Res 48:461-465

An SM, Koh J-S, Boo YC (2010) p-Coumaric acid not only inhibits human tyrosinase activity in vitro but also melanogenesis in cells exposed to UVB. Phytother Res 24:1175-1180

Charlwood BV, Charlwood SK, Molina-Tores JM (1990) Accumulation of secondary compounds by organized plant cultures. In: Charlwood BV, Rhodes MJC (eds) Secondary products from plant tissue cultures. Clarendon Press, Oxford, pp 167-200

Chlopčikova Š, Psotová J, Miteková P, Soušek J, Lichnovský V, Šimánek V (2004) Chemoprotective effect of plant phenolics against anthracycline-induced toxicity on rat cardiomyocytescaffeic, chlorogenic and rosmarinic acids. Phytother Res 18: 408-413

Chong KP, Rossall S, Atong M (2009) In vitro antimicrobial activity and fungitoxicity of syringic acid, caffeic acid and 4-hydroxybenzoic acid against Ganoderma boninense. J Agr Sci 1:15-20

De Graff WG, Myers LS, Mitchell JB, Hahn SM (2003) Protection against Adriamycin cytotoxicity and inhibition of DNA topoisomerase II activity by 3,4-dihydroxybenzoic acid. Int J Oncol 23:159-163

Ekiert H (1993) Ammi majus L (Bishop's weed): In vitro culture and the production of coumarin compounds. In: Bajaj YPS (ed) Biotechnology in agriculture and forestry, Medicinal and Aromatic plants IV, Springer, Berlin\HeidelberglNew York, pp 1-17

Ekiert H, Czygan FC (2007) Secondary metabolites in in vitro cultures of Ruta graveolens L. and Ruta graveolens ssp. divaricata (Tenore) Gams. In: Ramawat KG, Merillon JM (eds) Biotechnology: secondary metabolites, plants and microbes, Science Publication, Jersey $\backslash$ Plymouth, pp 445-482

Ekiert H, Gomółka E (2000a) a) Coumarin compounds in Ammi majus L. callus cultures. Pharmazie 55:684-687

Ekiert H, Gomółka E (2000b) Furanocoumarins in Pastinaca sativa L. in vitro culture. Pharmazie 55:618-620

Ekiert H, Piekoszewska A, Szewczyk A, Baczyńska S, Kuś A (2008) Isolation and identification of selected phenolic acids from biomass of Ruta graveolens L. and Ruta graveolens ssp. divaricata (Tenore) Gams cultured in vitro. Centr Eur Cong Life Sci Eurobiotech, Kraków, Abstracts in: Acta Biochim Polon $55: 61$ 
Ekiert H, Szewczyk A, Kuś A (2009) Free phenolic acids in Ruta graveolens $\mathrm{L}$. in vitro culture. Pharmazie 64:694-696

Ekiert H, Kwiecień I, Szopa A (2013) Rosmarinic acid production in plant in vitro cultures. Pol J Cosmetol 16:49-58

Ferguson LR, Zhu S-T, Harris PJ (2005) Antioxidant and antigenotoxic effects of plant cell wall hydroxycinnamic acids in cultured HT-29 cells. Mol Nutr Food Res 49:585-593

Itoh A, Isoda K, Kondoh M, Kawase M, Kobayashi M, Tamesada M, Yagi K (2009) Hepatoprotective effect of syringic acid and vanillic acid on concanavalin a-induced liver injury. Biol Pharm Bull 32:1215-1219

Itoh A, Isoda K, Kondoh M, Kawase M, Watari A, Kobayashi M, Tamesada M, Yagi K (2010) Hepatoprotective effect of syringic acid and vanillic acid on $\mathrm{CCl}_{4}$-induced liver injury. Biol Pharm Bull 33:983-987

Jiang Y, Kusama K, Satoh K, Takayama E, Watanabe S, Sakagami H (2000) Induction of cytotoxicity by chlorogenic acid in human oral tumor cell lines. Phytomedicine 7:483-491

Kikowska M, Budzianowski J, Krawczyk A, Thiem B (2012) Accumulation of rosmarinic, chlorogenic and caffeic acids in in vitro cultures of Eryngium planum L. Acta Physiol Plant 34:2425-2433

Kokotkiewicz A, Jaremicz Z, Łuczkiewicz M (2010) Aronia plants: a review of traditional use, biological activities, and perspectives for modern medicine. J Med Food 13:255-269

Kong WK, Zhao YL, Shan LM, Xiao XH, Guo WY (2008) Thermochemical studies on the quantity-antibacterial effect relationship of four organic acids from Radix Isatidis on Escherichia coli growth. Biol Pharm Bull 31:1301-1305

Kono Y, Shibata K, Kodama Y, Sawa Y (1995) The suppression of the $\mathrm{N}$-nitrosating reaction by chlorogenic acid. Biochem $\mathrm{J}$ 312:947-953

Kulling SE, Rawel HM (2008) Chokeberry (Aronia melanocarpa)—a review on the characteristic components and potential health effects. Planta Med 74:1625-1634

Lin AN, Nakatsui T (1998) Salicylic acid revisited. Int J Dermatol 37:335-342

Linsmaier EM, Skoog F (1965) Organic growth factor requirements of tobacco tissue culture. Physiol Plant 18:100-127

Makri O, Kintzios S (2004) In vitro rosmarinic acid production: An update. In: Ramawat KG (ed) Biotechnology of medicinal plants: Vitalizer and therapeutic. Science Publ Inc, Enfiled Plymouth, pp 19-32

Murashige T, Skoog F (1962) A revised medium for rapid growth and bioassays with tobacco tissue cultures. Physiol Plant 15:473-497

Nakamura Y, Torikai K, Ohigashi H (2001) A catechol antioxidant protocatechuic acid potentiates inflammatory leukocyte-derived oxidative stress in mouse skin via a tyrosinase bioactivation pathway. Free Rad Biol Med 30:967-978

Olthof MR, Hollman PC, Katan MB (2001) Chlorogenic acid and caffeic acid are absorbed in humans. J Nutr 131:66-71

Oszmański J, Wojdyło A (2005) Aronia melanocarpa phenolics and their antioxidant activity. Eur Food Res Technol 221:809-813

Piekoszewska A, Baczyńska S, Ekiert H (2008) Free phenolic acids in stationary liquid culture of Ruta graveolens ssp. divaricata (Tenore) Gams. Centr Eur Cong Life Sci Eurobiotech, Kraków, Abstracts in: Acta Biochim Polon 55:57

Pugazhendhi D, Pope GS, Darbre PD (2005) Oestrogenic activity of p-hydroxybenzoic acid (common metabolite of paraben esters) and methylparaben in human breast cancer cell lines. J Appl Toxicol 25:301-309

Ramawat KG, Mathur M (2007) Factors affecting the production of secondary metabolites. In: Ramawat KG, Merillon JM (eds) Biotechnology: secondary metabolites, plants and microbes. Science Publ Inc, JerseylPlymouth, pp 59-102

Silmestad R, Torskangerpoll K, Nateland HS, Johannessen T, Giske NH (2005) Flavonoids from black chokeberries, Aronia melanocarpa. J Food Compos Anal 18:61-68

Sroka Z, Cisowski W (2003) Hydrogen peroxide scavenging antioxidant and anti-radical activity of some phenolic acids. Food Chem Toxicol 41:753-758

Szopa A, Ekiert H (2011) Lignans in Schisandra chinensis in vitro cultures. Pharmazie 66:633-634

Szopa A, Ekiert H (2012) In vitro cultures of Schisandra chinensis (Turcz.) Baill. (Chinese magnolia vine) - a potential biotechnological rich source of therapeutically important phenolic acids. Appl Biochem Biotechnol 166:1941-1948

Szopa A, Ekiert H (2013) Production of deoxyschizandrin and $\gamma$-schizandrin in shoot-differentiating and undifferentiating callus cultures of Schisandra chinensis (Turcz.) Baill. (Chinese magnolia vine). J Biotech. doi:10.1016/j.jbiotec.2013.03.010

Szopa A, Ekiert H, Muszyńska B (2013) Accumulation of hydroxybenzoic acids and other biologically active phenolic acids in shoot and callus cultures of Aronia melanocarpa (Michx.) Elliott (black chokeberry) Plant Cell Tiss Org Cult 113:323-329

Thiem B, Krawczyk A (2003) Ellagic acid in in vitro cultures of Rubus chamaemorus L. Herba Pol 49:202-208

Tian S, Nakamura K, Cui T, Kayahara H (2005) High-performance liquid chromatographic determination of phenolic compounds in rice. J Chromatogr A 1063:121-128

Zdunczyka Z, Frejnagela S, Wróblewska M, Juśkiewicz J, Oszmiański J, Estrellac I (2002) Biological activity of polyphenol extracts from different plant sources. Food Res Intern 35: $183-186$ 